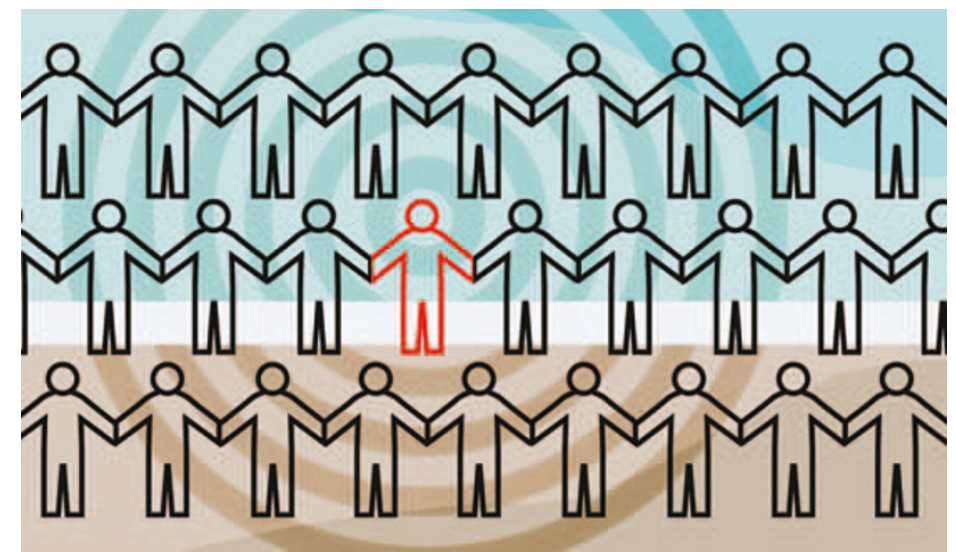

EPIDEMIOLOGY

\title{
Minority report
}

African-American women have a lower incidence of breast cancer compared with white women, but have a higher rate of breast cancer mortality. A prospective study by Rowan T. Chlebowski and colleagues finds that this is most likely explained by the aggressive nature of these cancers in a sizable proportion of African-

American women.

The authors wanted to address the limited attention within the literature given to differences in the distribution of breast cancer risk factors in ethnic minorities. To this end, they analysed data from 156,570 postmenopausal women participating in the Women's Health Initiative (WHI).

The authors' calculations included established risk factors such as current age, age at menarche, previous breast biopsies, age at first live birth and family history of breast cancer in first-degree relatives - factors that are all incorporated in the often-used Gail model of breast cancer risk. Other presumptive risk factors such as income level, educational level, alcohol intake and body-mass index (BMI) were also taken into account.

After a median follow-up of 6.3 years, 3,938 breast cancers were diagnosed. Age-adjusted incidences for all minority groups were lower than those in white women. However, adjustment for breast cancer risk factors accounted for all these differences except for the lower incidence and higher mortality evident in African Americans. The authors note that the tumours in this ethnic group were more often associated with a poor prognosis - they were high grade (poorly differentiated) and oestrogenreceptor negative. In fact, nearly one-third of all breast cancers in African-American women had this phenotype.

What lessons can be learned from this study? The authors propose that incorporating additional risk factors that vary with ethinicity into the widely used Gail model could provide a more accurate risk assessment in minority populations. Moreover, despite previous suggestions that reduced access to health care and a lower frequency of mammography were influencial in the outcome for AfricanAmerican women, the authors indicate that genetic risk factors are more likely to have an influencial role and these should be investigated further in larger, more comprehensive studies.

Oliver Childs

(2) References and links ORIGINAL RESEARCH PAPER Chlebowski, R. T. et al. Ethinicity and breast cancer: factors influencing differences in incidence and outcome. J. Natl Cancer Inst. 97, 439-448 (2005)

\section{TRIAL WATCH}

\section{Double whammy}

Recipients of solid-organ transplants are subject to continuous immunosuppressive treatment to prevent rejection. Although highly successful, such immunosuppressive regimens come at a price - an increased risk of cancer. Preliminary data now indicate that the immunosuppressive drug rapamycin (Sirolimus) might block both rejection and tumour development.

The incidence of Kaposi's sarcoma is 500 times greater in transplant recipients than in the general population. The main approach in treating Kaposi's sarcoma in these patients has been to reduce or stop their immunosuppressive drugs, but although the tumours regress, there is a significant risk of organ rejection.

In a study published in the New England Journal of Medicine, rapamycin has been used to treat 15 renal-transplant patients who developed Kaposi's sarcoma. All patients ( 12 men and 3 women) were checked and found to be negative for human immunodeficiency virus. The average duration for development of Kaposi's sarcoma lesions after transplant (confirmed by skin biopsy) was 12 months. At this point, treatment with the immunosuppressive agents cyclosporine and mycophenolate mofetil was stopped and replaced with rapamycin at a dose of 0.04-0.06 mg per kilogram of body weight per day.

Twelve of the patients showed evident tumour regression 1 month after rapamycin treatment was initiated, and by 3 months no lesions were evident in any of the patients. Skin biopsies at 6 months revealed complete tumour regression and, importantly, there were no incidences of acute rejection of the donated kidney. Lager controlled trials will be needed to address the efficacy of rapamycin in protecting transplant recipients against primarytumour development or tumour recurrence, and in maintaining a long-term immunosuppressive state.

ORIGINAL RESEARCH PAPER Stallone, G. et al. Sirolimus for Kaposi's sarcoma in renaltransplant recipients. N. Engl. J. Med. 352, 1317-1323 (2005)

\section{Jury still out on erythropoietin}

A meta-analysis published in the Journal of the National Cancer Institute finds that the treatment of cancer patients with erythropoietin to prevent complications arising from anaemia associated with cancer and cancer therapy might improve survival. These results contrast with two previously published large randomized controlled trials.

The meta-analysis focused on the efficacy of erythropoietin to prevent or treat anaemia with regard to blood transfusions, haematological response, adverse events and overall survival in studies - including randomized controlled trails - published between January 1985 and December 2001. Data analysed from 27 trials involving 3,287 adult patients indicated that erythropoietin treatment reduces the risk of blood transfusions and improves the haematological response in cancer patients. Suggestive, but not conclusive, results indicate that erythropoietin might improve overall survival rates. However, these findings will need to be verified against the background of published data indicating that erythropoietin increases the risk of thromboembolitic complications and concerns that it might stimulate tumour growth. ORIGINAL RESEARCH PAPER Bohlius, J. et al. Recombinant human erythropoietin and overall survival in cancer patients: results of a comprehensive meta-analysis. J. Nat/ Cancer Inst. 97, 489-498 (2005) 